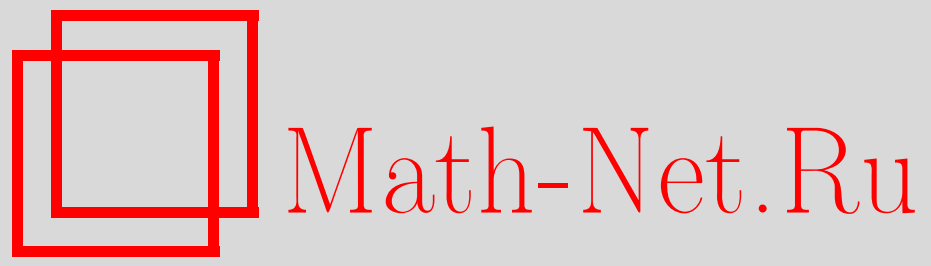

С. Ю. Оревков, Потенциал на бесконечности полиномиального образа круга, Функи. анализ и его прил., 1999, том 33, выпуск 4, 82-84

DOI: https://doi.org/10.4213/faa386

Использование Общероссийского математического портала MathNet.Ru подразумевает, что вы прочитали и согласны с пользовательским соглашением

http://www. mathnet.ru/rus/agreement

Параметры загрузки:

IP : 54.237 .206 .68

26 апреля 2023 г., 03:07:19

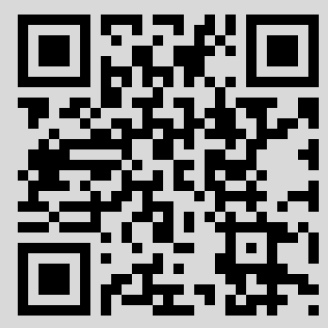


УДК 517.9

\title{
Потенциал на бесконечности полиномиального образа круга*
}

\author{
(C) 1999. С. Ю. Оревков
}

Пусть $D$ - область в $\mathbb{C}$, являющаяся образом $q(\Delta)$ единичного круга $\Delta$ при отображении $t \mapsto q(t)$, где

$$
q(t)=a_{0} t+a_{1} t^{2}+\cdots+a_{n} t^{n+1}, \quad a_{0} \in \mathbb{R},\left|a_{0}\right|>0,
$$

- многочлен, однолистный в $\Delta$.

Пусть $p(z)=\pi^{-1} \int_{D}(z-\zeta)^{-1} d \mu(\zeta)$ - потенциал области $D(d \mu(x+i y)=$ $d x d y)$. Обратная задача теории потенциала - это задача восстановления $D$ по ростку $p$ на $\infty$ (см. [1] и цитированную там литературу). При $|z| \gg 1$

$$
p(z)=\sum_{k \geqslant 0} \frac{c_{k}}{z^{k+1}}, \quad \text { где } c_{k}=\frac{1}{\pi} \int_{D} \zeta^{k} d \mu(\zeta)=\frac{1}{\pi} \int_{\Delta} q(t)^{k} q^{\prime}(t) \overline{q^{\prime}(t)} d \mu(t)
$$

$\left(c_{k}\right.$ - моменты области $\left.D\right)$. При помощи правой части $(2)$ можно задать $p(z)$ для любого многочлена $q(t)$ вида (1), не обязательно однолистного. Поскольку

$$
\frac{1}{\pi} \int_{\Delta} t^{k} \bar{t}^{m} d \mu(t)= \begin{cases}1 /(k+1) & \text { при } k=m, \\ 0 & \text { при } k \neq m,\end{cases}
$$

(2) позволяет выразить $c_{k}$ как многочлены от $a_{0}, a_{1}, \ldots, a_{n}, \bar{a}_{1}, \ldots, \bar{a}_{n}$ с рациональными коэффициентами. Из (2), (3) следует, что $c_{k}=0$ при $k>n$. Поэтому

$$
p(z)=c_{0} z^{-1}+c_{1} z^{-2}+\cdots+c_{n} z^{-(n+1)}, \quad c_{0} \in \mathbb{R},\left|c_{0}\right|>0 .
$$

Таким образом, мы получили полиномиальное отображение $\eta: V^{+} \rightarrow W^{+}$, где $V$ (соответственно $W$ ) есть векторное пространство над $\mathbb{R}$, изоморфное $\mathbb{R} \times \mathbb{C}^{n}$, с координатами $\left(a_{0}, \ldots, a_{n}\right)$ (соответственно $\left.\left(c_{0}, \ldots, c_{n}\right)\right), V^{+}$(соответственно $W^{+}$) есть полупространство $a_{0}>0$ (соответственно $\left.c_{0}>0\right)$ и $\eta$ задано формулой $\eta(q)=p$. Мы отождествляем точки полупространства $V^{+}$(соответственно $\left.W^{+}\right)$с многочленами $q$ вида (1) (соответственно вида (4)).

Обозначим через $j(\eta)$ якобиан отображения $\eta$ относительно форм объема $d a_{n} \wedge \cdots \wedge d a_{1} \wedge d a_{0} \wedge d \bar{a}_{1} \wedge \cdots \wedge d \bar{a}_{n}$ и $d c_{n} \wedge \cdots \wedge d c_{1} \wedge d c_{0} \wedge d \bar{c}_{1} \wedge \cdots \wedge d \bar{c}_{n}$. Для $q \in V$ определим $t_{1}, \ldots, t_{n}$ формулой $q^{\prime}(t)=\prod_{i=1}^{n}\left(1-t_{i} t\right)$.

Teоpema. $j(\eta)=2 a_{0}^{n^{2}+n+1} \operatorname{Res}_{t}\left(t^{n} \bar{q}^{\prime}\left(t^{-1}\right), q^{\prime}(t)\right)=2 a_{0}^{n^{2}+3 n+1} \prod_{i, j=1}^{n}\left(1-t_{i} \bar{t}_{j}\right)$.

ЗАмечАниЕ (П. Этингоф). Похожая формула встречается в конформной теории поля как формула для коцикла, задающего центральное расширение комплексификации группы $\operatorname{Diff}\left(S^{1}\right)$.

Пусть $\mathscr{I}_{n}=\left\{q \in V^{+} \mid q^{\prime}\right.$ не имеет корней в $\left.\Delta\right\}$.

СлЕДСТВИЕ 1 (см. [1]). Оораничение $\eta$ на Int $\mathscr{I}_{n}$ - погружение.

СлЕДСТвиЕ 2. Замыканием складки отображения $\eta$ является (вещественная) гиперповерхность $F=\left\{q \in V^{+}\left|\exists t_{i}, q^{\prime}\left(t_{i}\right)=0 u\right| t_{i} \mid=1\right\}$.

*Работа выполнена при частичной поддержке грантов РФФИ-96-01-01218 и DGICYT SAB95-0502. 
СЛЕДСТВИЕ 3. Замыканием множества ветвления отображения $\eta$ является подмногообразие коразмерности два $B=\left\{q \in V^{+} \mid \exists t_{i} \neq t_{j}, t_{i} \bar{t}_{j}=1\right\}$.

ДокАЗАТЕЛЬСтво ТЕоремЫ. Обозначим $\partial / \partial a_{m}$ через $\partial_{m}$ и $\partial / \partial \bar{a}_{m}$ через $\bar{\partial}_{m}, m=0, \ldots, n\left(\partial_{0}=\bar{\partial}_{0}\right)$. Положим

$$
J=\left(\begin{array}{ccccccc}
\bar{\partial}_{n} c_{n} & \ldots & \bar{\partial}_{1} c_{n} & \partial_{0} c_{n} & \partial_{1} c_{n} & \ldots & \partial_{n} c_{n} \\
\ldots \ldots \ldots \ldots \ldots \ldots \ldots \ldots \ldots \ldots & \ldots \ldots \ldots \\
\bar{\partial}_{n} c_{1} & \ldots & \bar{\partial}_{1} c_{1} & \partial_{0} c_{1} & \partial_{1} c_{1} & \ldots & \partial_{n} c_{1} \\
\bar{\partial}_{n} c_{0} & \ldots & \bar{\partial}_{1} c_{0} & \partial_{0} c_{0} & \partial_{1} c_{0} & \ldots & \partial_{n} c_{0} \\
\bar{\partial}_{n} \bar{c}_{1} & \ldots & \bar{\partial}_{1} \bar{c}_{1} & \partial_{0} \bar{c}_{1} & \partial_{1} \bar{c}_{1} & \ldots & \partial_{n} \bar{c}_{1} \\
\ldots \ldots \ldots & \ldots & \ldots \ldots \ldots \ldots \ldots \ldots \ldots \\
\bar{\partial}_{n} \bar{c}_{n} & \ldots & \bar{\partial}_{1} \bar{c}_{n} & \partial_{0} \bar{c}_{n} & \partial_{1} \bar{c}_{n} & \ldots & \partial_{n} \bar{c}_{n}
\end{array}\right)
$$

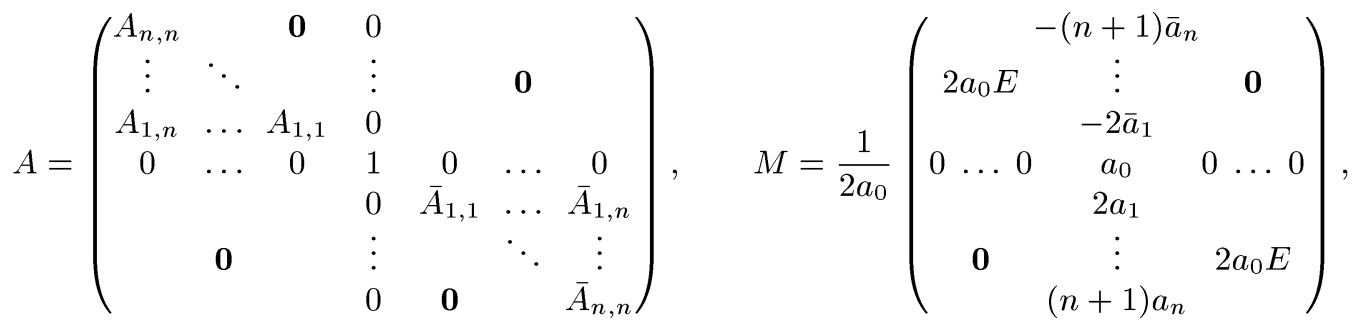

где $A_{k, j}, j \geqslant k>1$, - многочлены от $a_{0}^{ \pm 1}, a_{m}, \bar{a}_{m}$, такие, что $\sum_{j \geqslant k} A_{k, j} q^{j}=t^{k}$. Вычислим $A J$. Положим $A_{k, j}=0$ при $j<k, A_{0,0}=1, A_{0, j}=0$ при $j>0$ и $a_{m}=0$ вне $0 \leqslant m \leqslant n$. Обозначим $\pi^{-1} \int_{\Delta} f(t) \overline{g(t)} d \mu(t)$ через $\langle f, g\rangle$. Тогда $(3)$ означает $\left\langle t^{k}, t^{m}\right\rangle=\delta_{k m} /(k+1)$. Заметим, что $\partial_{m} q=t^{m+1}, \partial_{m} q^{\prime}=(m+1) t^{m}$ и

$$
\left(\partial_{m} q^{j}\right) q^{\prime}=\frac{d q^{j}}{d q} \partial_{m} q \cdot q^{\prime}=\frac{d q^{j}}{d q} \frac{d q}{d t} \partial_{m} q=\frac{d q^{j}}{d t} \partial_{m} q
$$

Следовательно, при всех $m \geqslant 0$ мы имеем

$$
\begin{aligned}
\sum_{j} A_{k, j}\left\langle\partial_{m}\left(q^{j} q^{\prime}\right), q^{\prime}\right\rangle & =\left\langle\left(\partial_{m} q\right) \cdot \frac{d}{d t} \sum A_{k, j} q^{j}, q^{\prime}\right\rangle+\left\langle\left(\partial_{m} q^{\prime}\right) \cdot \sum A_{k, j} q^{j}, q^{\prime}\right\rangle \\
& =\left\langle t^{m+1} \cdot k t^{k-1}, q^{\prime}\right\rangle+\left\langle(m+1) t^{m} \cdot t^{k}, q^{\prime}\right\rangle \\
& =(m+k+1)\left\langle t^{m+k}, q^{\prime}\right\rangle=(m+k+1) \bar{a}_{m+k}, \\
\sum_{j} A_{k, j}\left\langle q^{j} q^{\prime}, \partial_{m} q^{\prime}\right\rangle & =\left\langle q^{\prime} t^{k},(m+1) t^{m}\right\rangle=(m-k+1) a_{m-k} .
\end{aligned}
$$

Поэтому элементы верхнего правого квадранта в $A J$, верхнего левого квадранта и верхней части среднего столбца равны соответственно

$$
\begin{array}{rlrl}
\sum A_{k, j} \partial_{m} c_{j} & =\sum A_{k, j}\left\langle\partial_{m}\left(q^{j} q^{\prime}\right), q^{\prime}\right\rangle=(m+k+1) \bar{a}_{m+k}, \quad & m>0, \\
\sum A_{k, j} \bar{\partial}_{m} c_{j} & =\sum A_{k, j}\left\langle q^{j} q^{\prime}, \partial_{m} q^{\prime}\right\rangle=(m-k+1) a_{m-k}, & m>0, \\
\sum A_{k, j} \partial_{0} c_{j} & =\sum A_{k, j}\left(\left\langle\partial_{0}\left(q^{j} q^{\prime}\right), q^{\prime}\right\rangle+\left\langle q^{j} q^{\prime}, \partial_{0} q^{\prime}\right\rangle\right)=(k+1) \bar{a}_{k}-(k-1) a_{-k} \cdot
\end{array}
$$


Каждый элемент нижней части матрицы $A J$ сопряжен центрально симметричному элементу верхней части. Значит,

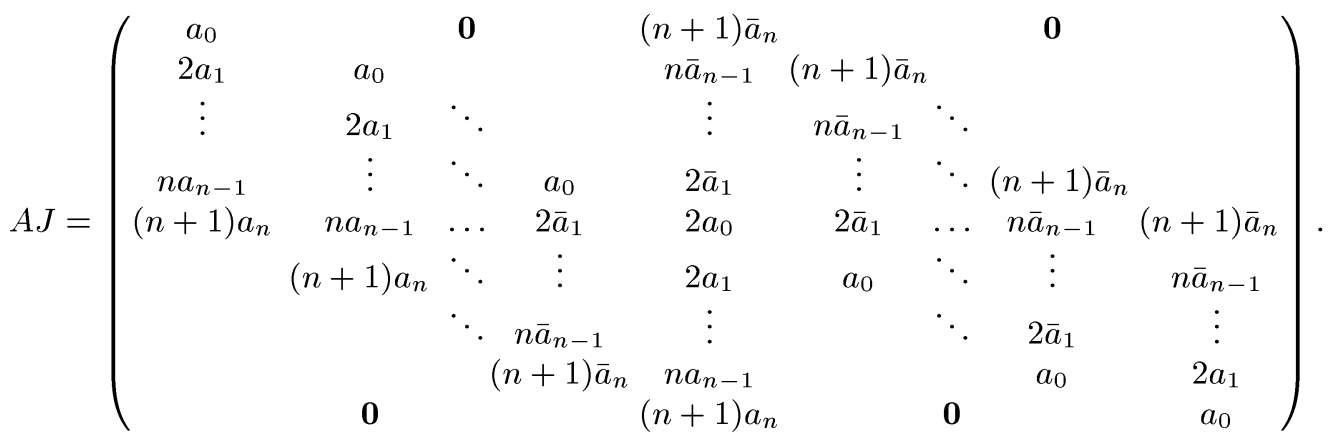

Умножая $A J$ справа на $M$, мы заменяем средний столбец на $\left(0, \ldots, 0, a_{0}, 2 a_{1}, \ldots\right.$, $\left.(n+1) a_{n}\right)^{t}$ и получаем матрицу с верхней строкой $\left(a_{0}, 0, \ldots, 0\right)$, и дополнительный минор к $a_{0}$ - транспонированная матрица Сильвестра для результанта многочленов $a_{0}+2 a_{1} t+\cdots+(n+1) a_{n} t^{n}$ и $(n+1) \bar{a}_{n}+n \bar{a}_{n-1} t+\cdots+\bar{a}_{0} t^{n}$. Значит, $\operatorname{det} A \operatorname{det} J \operatorname{det} M=a_{0} \operatorname{Res}_{t}\left(q^{\prime}, t^{n} \bar{q}^{\prime}\left(t^{-1}\right)\right)$.

Ясно, что $\operatorname{det} M=\frac{1}{2}$ и $A_{k, k}=a_{0}^{-k}$, а следовательно, $\operatorname{det} A=\prod A_{k, k}^{2}=a_{0}^{-n(n+1)}$. Осталось заметить, что обращение порядка элементов $\partial a_{m}$ в $J$ меняет знак таким же образом, как и перестановка аргументов результанта.

Я благодарю П. Этингофа и Н. Г. Кружилина за полезные обсуждения.

\title{
ЛиТЕРАТУРА
}

1. Прилепко А. И. Потенциала теории обратные задачи. Математическая энциклопедия. Т. 4, Советская энциклопедия, 1984, с. 520-523.

\section{О разнообразии отображений Пуанкаре для кубических уравнений с переменными коэффициентами*}

\author{
(C) 1999. А. А. ПАнов
}

1. Введение. Основным объектом нашего изучения является уравнение вида

$$
\dot{x}=a_{3}(t) x^{3}+a_{2}(t) x^{2}+a_{1}(t) x+a_{0}(t), \quad t \in[0,1], a_{i} \in C^{1} .
$$

Введем несколько стандартных обозначений. Рассмотрим уравнение вида

$$
\dot{x}=h(x, t), \quad h \in C^{1} .
$$

*Работа частично поддержана грантами РФФИ 95-01-01258, CRDF RM1-229, INTAS 93-05-07. 